\title{
Mental health interventions and future major depression among primary care patients with subthreshold depression
}

Authors:

Sandra K. Davidson.

Department of General Practice

University of Melbourne, Australia

Meredith G. Harris

School of Population Health

The University of Queensland, Australia

Christopher F. Dowrick

University of Liverpool, UK

Caroline A. Wachtler

Department of General Practice

University of Melbourne, Australia

Centre for Family Medicine

Karolinska Institute, Sweden

Jane Pirkis

Melbourne School of Population and Global Health

University of Melbourne, Australia

Jane M. Gunn

Department of General Practice

University of Melbourne, Australia

Corresponding author:

Dr Sandra Davidson

Department of General Practice

University of Melbourne

200 Berkeley Street,

Carlton, Vic. 3053

Australia

Ph: +6138344 7276

Fax: +61393476135

Email:sdav@unimelb.edu.au 


\begin{abstract}
Background

Subthreshold depression is prevalent in primary care and is associated with poorer quality of life, higher health care use and increased risk of major depressive disorder (MDD). Currently, it is unclear how subthreshold depression should be managed in primary care and no studies have investigated the relationship between current models of care and the development of MDD. This study aimed to describe usual care over a six month follow-up for primary care patients with subthreshold depression and to investigate the relationship between usual care and the development of MDD.
\end{abstract}

\title{
Methods
}

Data were derived from 250 participants with subthreshold depression from the diamond study, a longitudinal cohort study of primary care patients. Participants completed questionnaires at three and six months on their health care use, the interventions they received and their depression status. Interventions were categorised according to the NICE guidelines for the management of depression in adults. Generalised estimating equation (GEE) models and logistic regression were used to estimate the association between receiving an intervention and MDD over six months.

\section{Results}

Four fifths $(80.8 \%)$ of participants received a mental health intervention. Therapeutic listening, reassurance, pharmacotherapy and advice to exercise were most common. Subsequent MDD was predicted by history of depression, baseline depressive symptom severity and receiving a mental health intervention.

\section{Limitations}

Usual care was assessed via patient self-report.

\section{Conclusions}

Primary care physicians deliver mental health interventions to most subthreshold patients.

However, it appears that current interventions are not averting MDD. Further research to identify effective interventions which are feasible in primary care is needed.

Keywords: subthreshold depression; major depressive disorder; primary care; treatment; usual care 


\section{Introduction}

Subthreshold depression, where depressive symptoms are present but do not meet standard diagnostic criteria for major depressive disorder (MDD) (National Institute for Health \& Clinical Excellence, 2010), is prevalent in primary care. Estimates of prevalence range from 9\% in health maintenance organisations (Simon and Von Korff, 1995 ) to 35\% in a university-based primary care practice (Wagner et al., 2000). The subthreshold population is heterogeneous and includes people in partial remission from a major depressive episode, people with persistent subthreshold symptoms and people with recent onset of symptoms (Druss et al., 2007). Compared to people with no depressive symptoms, those with subthreshold depression have significantly higher functional impairment (Wagner et al., 2000) and are at increased risk of developing MDD (Cuijpers and Smit, 2004; Pietrzak et al., 2013). Moreover, people with subthreshold depression and a history of depression are at significantly higher risk of MDD than people with subthreshold depression and no history of depression (Karsten et al., 2011). At the same time, there is a high natural recovery rate with approximately $70 \%$ of the subthreshold population not progressing to MDD (Hermens et al., 2004; Pietrzak et al., 2013).

There are two schools of thought as to how health professionals should respond to subthreshold depression (Ackermann and Williams, 2002). On the one hand, proponents of treatment draw on the small number of psychological trials showing that treating subthreshold depression in primary care has the potential to avert new cases of major depressive disorder (MDD) (Cuijpers et al., 2014; Cuijpers et al., 2007; Morgan et al., 2012; Willemse et al., 2004), albeit with high numbers needed to treat (NNT) (16 in Willemse et al (2004) and 25 in Morgan et al (2012)). On the other hand, the high rate of natural recovery (Hermens et al., 2004; Pietrzak et al., 2013), combined with large NNT, suggests that treating subthreshold depression may subject many people who have normal, self-remitting emotional distress to unnecessary, burdensome and potentially stigmatising treatment (Dowrick and Frances, 2013). It is further argued that providing intensive treatments to people with subthreshold depression may represent a misallocation of scarce resources with interventions diverted toward those with the least need, thus contributing to the treatment gap for those with a diagnosable mental disorder (Druss et al., 2007; Wang et al., 2005).

In 2009, the NICE guidelines included the management of persistent subthreshold depressive symptoms for the first time. However, they acknowledge that the empirical evidence supporting these recommendations is very limited (National Institute for Health \& Clinical 
Excellence, 2010). Currently, the guidelines recommend a stepped care approach beginning with general sleep hygiene advice and, if necessary, progressing to active monitoring which includes discussing the presenting problem, providing psychoeducation and conducting follow-up. For people with continued symptoms, low intensity psychosocial interventions such as a group exercise programme, a peer support group, guided self-help based on the principles of cognitive behavioural therapy (CBT) or computerised CBT are recommended. If symptoms still persist, high intensity psychosocial interventions comprising multiple sessions of CBT or Interpersonal Therapy (IPT) are recommended. Antidepressants are only recommended for people with persistent (i.e. more than two years) symptoms or for those whose symptoms persist after other interventions (National Institute for Health \& Clinical Excellence, 2010).

The caveat in the NICE guidelines is that the evidence base on the effectiveness of psychological treatments is too small to support clear recommendations. This caveat underpins their subsequent call for further research to examine the efficacy of counselling and low intensity CBT compared to usual care in the treatment of subthreshold depression. Of course, this suggests that the nature of usual care for subthreshold depression is known. In fact, no studies have examined usual care in the primary care setting for people with subthreshold depression or investigated the relationship between usual care and depression outcomes. The current study aims to address this gap by describing: the proportion of primary care attendees with subthreshold depression who received a mental health intervention over six months; the type of intervention they received and the relationship between receiving an intervention and subsequent development of MDD. In order to estimate whether an individual has persistent symptoms, and is more likely to need the interventions described later in the stepped care model, we separate people with subthreshold depression into those with a history of depression and those without.

\section{Method}

\section{Procedure}

Data were derived from an ongoing longitudinal cohort study: Diagnosis, Management and Outcomes of Depression (diamond) (Gunn et al., 2008). diamond is designed to document the experiences, service use, treatment and health outcomes of primary care patients with a broad range of severity of depressive symptoms. Its rationale and methods have been described previously (Gunn et al., 2008). Assessments consist of written questionnaires and telephone interviews. The present study reports data from baseline, three and six months. A six month 
follow-up was chosen as we considered that this was a reasonable time frame for GPs to respond to patients with subthreshold symptoms. Exclusion criteria for the study were: unable to read English; presence of a terminal illness, not residing in the community and outside the age range of 17 - 76 years.

\section{Participants}

A list of 200 randomly selected GPs, stratified by population distribution to ensure a representative rural and metropolitan sample and who had provided at least 1,500 consultations in the previous year, was provided to the researchers by the Australian Health Insurance Commission. Thirty GPs from this list, all from different practices, agreed to participate in the study. Prior to patient recruitment, the GPs completed a questionnaire describing their practice setting, mental health education or skills training and areas of interest.

Between 2005 and 2006, 17,780 patients aged between 18 and 75 years listed with the 30 study GPs were sent a screening survey which included the Centre for Epidemiologic Studies Depression Scale (CESD) (Radloff, 1977). A total of 7,509 (42.2\%) people returned a completed survey, 1,793 (23.9\%) of whom scored $\geq 16$ on the CESD, indicating the presence of depressive symptoms. The prevalence of $24 \%$ for depressive symptoms is consistent with other studies of primary care practices (Gilchrist and Gunn, 2007) which suggests that the practices in diamond are representative in regards to the proportion of patients with depressive symptoms. People with a score of $\geq 16$ on the CESD were invited to participate in diamond and 789 (44.0\%) agreed.

At baseline, all patient participants completed the questionnaire administered Primary Health Questionnaire depression module (PHQ-9) (Kroenke et al., 2001) and 726 (92\%) completed the telephone administered Composite International Diagnostic Interview (CIDI) (Kessler and Ustun, 2004). Of the 789 participants who completed the PHQ-9, 211 (26.7\%) met the criteria for major depressive disorder using the algorithm scoring method to assess the presence of major depression in the past two weeks. Of the 726 who completed the CIDI, 370 $(51.0 \%)$ met the criteria for major depression over the past 12 months (Kessler and Ustun, 2004). In total, $494(62.6 \%)$ participants met the criteria for major depressive disorder on either the PHQ-9 or the CIDI and were excluded from the present study. The remaining 295 participants were classified as having subthreshold depression.

The sensitivity of the algorithmic scoring method of the PHQ-9 is rather low at 0.77 (Wittkampf et al., 2007) and may miss some patients with depressive disorder. On the other hand, 
the CIDI has been shown to have a high false positive rate, generating a diagnosis of depression when a true case of depression does not exist (Kurdyak and Gnam, 2005). By using both the PHQ-9 and the CIDI to identify probable depression it is unlikely that participants classified as having subthreshold depression were true cases of depression.

Of the subthreshold group, $250(84.7 \%)$ completed surveys at three and six month followup. Of these, $118(47.2 \%)$ had previously been told by a doctor or a psychologist they had depression and were considered to have a history of depression. The remaining 132 (52.8\%) reported never having been told by a doctor or psychologist that they had depression and were classified as not having a history of depression. Participant's scores on the PHQ-9 and CIDI were not disclosed to GPs.

\section{Measures}

\section{Baseline sociodemographic and clinical characteristics}

Information on a range of sociodemographic and clinical characteristics were collected at study entry including gender, age, education, employment status, reliance on a government pension, marital status, living arrangements, baseline depression severity, chronic illness, life events, smoking status, hazardous alcohol consumption, anxiety disorder and suicidal ideation. The PHQ-9 continuous score was used to indicate baseline depression severity (Kroenke et al., 2001). Chronic illness was assessed by asking participants if, in the past 12 months, they had any one of the 11 most commonly managed conditions in Australian primary care (i.e. asthma, emphysema, diabetes, arthritis, back problem, chronic sinusitis, high cholesterol, heart disease, cancer, stroke or dermatitis) (Britt et al., 2007; Gunn et al., 2012). Participants were considered to have a chronic illness if they answered yes to any of these conditions. Life events were measured using a life events questionnaire (Norbeck, 1984) adapted from the Life Experiences Scale (Sarason et al., 1978). Anxiety symptoms were measured using the anxiety module of the PHQ-9 which includes seven items based on DSM-IV criteria for generalised anxiety disorder (Spitzer and Kroenke, 1999). Suicidal ideation was assessed using item nine of the PHQ-9 which asks whether, in the last two weeks, the person has been bothered by thoughts that they would be better off dead or of hurting themselves in some way. Participants were considered to have suicidal ideation if they indicated they felt this way several days or more in the last two weeks. A pattern of hazardous drinking over the past 12 months was assessed using the four item Fast Alcohol Screening Test (FAST) (Hodgson et al., 2002). 


\section{Visits to GPs and other health professionals}

At three and six month follow-up, participants completed questionnaires asking how often in the previous three months they saw a GP and the number of different GPs they saw. Participants were also asked whether they attended a psychologist, counsellor or psychiatrist at least once. Participants were deemed to have seen a psychologist, counsellor or psychiatrist if they responded 'yes' at either follow-up.

\section{Mental health interventions}

At three and six months, participants were asked what interventions they had received from GPs for their emotional well-being and what self-help interventions they had used (Gunn et al., 2008). To help organise our understanding of the provision of services for people with subthreshold depression we mapped the available data items against the stepped care recommendations for managing adults with depression in the NICE guidelines (National Institute for Health \& Clinical Excellence, 2010) (Box 1). Receipt of sleep hygiene advice was evidenced if participants reported that a GP had provided them with advice on sleep and wake times, diet, exercise or drug and alcohol use. Participants were considered to have received active monitoring if they reported that they had seen a GP at least once in the first three month follow-up and a GP gave them a chance to talk about how they were feeling, or a GP provided reassurance, encouragement and explanation, or a GP provided them with leaflets, booklets or videos about depression. The criterion of only one follow-up visit in the first three months was chosen because it was considered that for some people, their symptoms may have completely resolved and therefore further monitoring visits would not be indicated. Receipt of a low intensity psychosocial intervention was measured by asking participants if they had attended a self-help group for emotional well-being, or if they had received counselling from a GP in relation to emotional well-being, or were helped to talk through their problems by a GP, or had a series of scheduled visits for emotional well-being or had used an educational or therapeutic website for depression, stress or worries. Estimates of high-intensity psychosocial interventions were derived by combining items on the receipt of counselling from a GP or being talked through their problems by a GP with receiving at least 16 visits to a GP over six months. Sixteen visits were selected as the minimum threshold as it aligns with the NICE recommendations for high-intensity psychosocial interventions. These recommendations are based on the evidence from the majority of CBT and IPT trials which include 16-20 sessions over a six month period (National Institute for Health \& Clinical Excellence, 2010). Receipt of antidepressant therapy was evidenced if 
participants were prescribed an antidepressant by a GP during follow-up. We also asked whether participants were referred by a GP to another health professional for emotional well-being.

\section{Major depressive disorder}

Participants completed the PHQ-9 at three and six months. Presence of MDD in the past two weeks was determined using the algorithm scoring method (Kroenke et al., 2001).

\section{Statistical analysis}

The analysis began by using percentages to describe the characteristics of the primary care setting from which participants were recruited. Next, descriptive statistics were used to profile study participants' sociodemographic and clinical characteristics, health care use, interventions received and occurrence of MDD. Regression models were fitted using generalised estimating equations (GEE) with robust standard errors to 1) compare participants with a history of depression to those with no history of depression, and 2) compare participants who did develop MDD with those who did not. The GEE model takes into account the within cluster correlations that may arise from recruiting multiple patients from each of the 30 primary care clinics. Finally, univariate logistic regression analysis was used to estimate the association between receiving an intervention and MDD over six months and multiple logistic regression was used to assess this association when history of depression and baseline depression severity was taken into account.

\section{Results}

\section{Primary care setting}

All GP practices from which patients were recruited were private, rather than corporate, practices and $67 \%$ of the 30 GPs were either partners or principals in the practice. Eight (27\%) GPs reported that they had a special interest in mental health and 17 (57\%) reported spending at least five hours on education or skills training for depression in the past 12 months.

\section{Sample characteristics}

The average age of participants at baseline was 50 years (Table 1$)$. The majority $(n=175$; $70.0 \%$ ) were women; 118 (47.4\%) were married and 50 (20.0\%) lived alone. A government pension was the main source of income for 95 (38.0\%) participants and 35 (14.1\%) reported leaving school before Year 10. Of the 250 participants, 175 (70.0\%) reported having a chronic physical illness, $61(24.5 \%)$ were current smokers and $64(25.7 \%)$ drank alcohol at hazardous 
levels. Suicidal ideation was reported by 49 (19.7\%) participants and 40 (16.2\%) met the PHQ-9 criteria for anxiety disorder.

People with a history of depression had a small, but statistically significant, increase in depressive symptoms at baseline compared to people with no history of depression. A history of depression was also significantly associated with being female, relying on a pension and living alone.

\section{Visits to GPs and other health professionals}

Twenty-one (8.4\%) participants did not visit a GP during the follow-up period. The average number of GP visits over six months was 4.7 with a range of 0-24 (Table 2). Participants visited an average of 2.1 different GPs, with eight different GPs representing the upper range. Only 16 people $(6.4 \%)$ visited a psychiatrist during follow-up and 27 people (10.8\%) visited a psychologist or counsellor. A history of depression was associated with significantly more visits to a GP and an increased likelihood of visiting a psychiatrist during follow-up.

\section{Interventions received}

The odds of participants with a history of depression receiving a mental health intervention was 3.2 compared to those with no history of depression ( $88.7 \%$ vs. $71.4 \%)$. Sleep hygiene advice was received by 98 (42.8\%) participants (Table 3). Advice regarding exercise was the most common with 25 people (10.0\%) reporting that this was the only type of sleep hygiene advice they received. Despite more than a quarter of participants reporting they drank at hazardous levels, advice on reducing alcohol or drugs was the least common. One person $(0.4 \%)$ reported they received advice only on drug or alcohol reduction.

Of all the interventions, participants were most likely to receive active monitoring. The odds of receiving reassurance, the chance to talk or psychoeducation was two times greater for participants with a history of depression compared to those without. However, 19\% of both groups failed to meet the criteria of at least one follow-up GP visit and there was no overall difference in the odds of receiving active monitoring.

Seventy-five participants (30.0\%) received a low intensity psychosocial intervention and only two $(0.9 \%)$ received a high intensity psychosocial intervention (comprising a CBT or IPT plus 16 or more GP visits). The odds of receiving a prescription for antidepressants during the six month follow-up were more than five times greater for people with a history of depression 
(39.7\%) compared to people with no history of depression (11.4\%). Only $14(6.1 \%)$ participants were referred to a mental health specialist.

\section{Major depressive disorder}

Of the 250 participants with subthreshold depression at baseline, 45 (18.0\%) met the criteria for MDD at least once over the following six months. Among people with a history of depression, 33 (25.0\%) developed MDD and among people with no history of depression 12 (10\%) developed MDD. Figure 1 shows that the percentage of participants with MDD declined slightly between three and six months for people with no history of depression (from $6.8 \%$ to $5.1 \%$ ). In contrast, the proportion of participants with MDD increased sharply (from $10.6 \%$ to $18.9 \%$ ) among people with a history of depression.

As shown in Table 4, people who developed MDD had significantly more depressive symptoms at baseline and were two and half times more likely to have a history of depression. None of the other patient characteristics examined in this study showed a statistically significantly association with future MDD.

\section{Mental health interventions and major depression}

Because of the very small numbers of participants who received a high intensity psychosocial intervention it was not possible to analyse the relationship between this form of care and developing MDD. In the univariate analysis, three out of the remaining five interventions were significantly associated with MDD over six months (Table 5). People who received sleep hygiene advice, active monitoring or pharmacotherapy were more likely to develop MDD during follow-up. When the analysis was adjusted to account for history of depression and baseline depression severity, the association between active monitoring and MDD was no longer significant. The associations between sleep hygiene advice and MDD, and the association between pharmacotherapy and MDD, remained statistically significant in the adjusted analysis but were considerably weaker.

\section{Discussion}

\section{Main findings}

This is the first study to prospectively examine the usual care received by primary care attendees with subthreshold depression and to investigate the relationship between different levels of usual care and the subsequent occurrence of MDD. A number of key findings arise from 
this study. We found that a small but substantial proportion of people with subthreshold depression did not receive any mental health response from a GP. If participants did receive a response it was most likely to be at the lifestyle advice and support end of the spectrum rather than at the intensive psychosocial intervention end. Contrary to the guidelines on the management of subthreshold depression (1), antidepressants were prescribed for more than ten percent of people with no history of depression and for forty percent of people with a history of depression.

Over 80 percent of primary care patients with subthreshold depression did not develop MDD over six months. People who did develop MDD had significantly higher depressive symptom severity at baseline and were more likely to have a history of depression. Unexpectedly, univariate analysis found that receiving some mental health interventions was also associated with an increased likelihood of developing MDD. However, these associations were either no longer significant, or considerably weakened, when history of depression and baseline depression severity was taken into account. This suggests that GPs are identifying variations in depressive symptom severity, even in subthreshold patients, and are responding by providing higher levels of care accordingly.

\section{Comparisons with the literature}

Consistent with previous studies, we found that people with subthreshold depression are heavy users of primary care services, attending on average four to five times over six months (Goldney et al., 2004; Wagner et al., 2000). This rate of attendance is double the national average which is approximately 4.6 visits to a GP over 12 months (Medicare Australia Statistics). Despite their frequent presence in primary care, there are no previous studies describing usual care for subthreshold depression against which we can compare our results. Instead, we draw on studies examining mental health interventions for primary care patients with mild major depression (Druss et al., 2007; Harris et al., In press). These studies report a similar pattern to ours, where a high proportion of people receive interventions that may be potentially beneficial, such as therapeutic listening or reassurance, but very few receive interventions that meet the criteria for CBT or IPT and even fewer receive CBT or IPT over multiple sessions. However, whereas studies investigating treatment rates for MDD conclude that low levels of CBT or IPT interventions indicate that depression is not being treated adequately by primary care doctors, the same conclusion cannot be drawn here because the evidence regarding adequate treatment for subthreshold depression is not yet established (National Institute for Health \& Clinical 
Excellence, 2010). Indeed, in this naturalistic cohort study we did not find any intervention to be associated with a reduction in the occurrence of MDD.

Our finding that $10 \%$ of people with no history of depression and $25 \%$ of people with a history of depression developed MDD over six months is very similar to the rates of $7 \%$ and $23 \%$ respectively, reported by Karsten et al (2011). In combination, these studies indicate that a history of depression may be a reliable prognostic factor for the development of MDD among people with subthreshold symptoms. These findings, as well as our finding that symptom severity was significantly associated with developing MDD, extend the 2004 review by Hermens et al who found no studies examining prognostic factors for MDD among the subthreshold population (Hermens et al., 2004).

\section{Implications}

The findings of this study have implications for the future development of guidelines for the management of subthreshold depression. Our results support distinguishing the subthreshold population into those with a history of depression and those with no history of depression. Given that $90 \%$ of people with subthreshold symptoms and no history of depression did not develop MDD over six months suggests that, for people with recent onset of symptoms, the risk associated with labelling and medicalising these symptoms may outweigh the benefits that can be gained from intensive interventions (Dowrick and Frances, 2013). Therefore, the watch and wait approach implicit in the first two steps of the NICE guidelines appears to be appropriate for people with recent onset of symptoms and no history of depression.

In contrast, although $75 \%$ of people with a history of depression also did not develop MDD over six months, the risks compared to the benefits of treatment moves slightly toward favouring intervention, at least theoretically. We say theoretically, because in this naturalistic cohort study we failed to find evidence of the benefits of intervening. In contrast, receiving sleep hygiene advice and pharmacotherapy were both associated with increased likelihood of MDD over six months. However, we consider that the relationship between receiving an intervention and developing MDD is not because these types of usual care are causally involved in the development of MDD. Instead, this relationship is likely to reflect confounding by severity which occurs when clinicians provide higher levels of intervention to those with the greatest need (Salas et al., 1999).

Overall, GPs appear to be identifying people most at risk of MDD and are providing them with more intensive support. However, it would seem that they are either not providing the 
appropriate intervention, or are not providing a sufficient dose of the appropriate intervention, to prevent the onset of MDD. Our finding that many people with subthreshold depression were prescribed an antidepressant suggests that non-evidence based interventions are frequently being delivered. In particular, it is possible that pharmacotherapy is being delivered at the expense of more appropriate interventions like sleep hygiene advice and psychoeducation.

Finally, we found that social disadvantage is higher among people with a history of depression, a factor which may work to perpetuate their depressive symptoms, affect their ability to respond positively to treatment and impose additional barriers to full recovery. Guidelines should consider how to incorporate these social determinants into recommendations for the management of subthreshold depression.

\section{Further research}

Further research focusing on the development of a prognostic tool which GPs can use to identify risk of MDD in subthreshold patients would be valuable. Such a tool could reduce over treatment of a large proportion of people who do not need treatment and focus attention on those that could benefit. This research should run parallel with research to identify interventions that prevent the onset of MDD among people with subthreshold depression and that GPs can feasibly incorporate into their usual care. The current study shows that interventions requiring intensive CBT or IPT over multiple sessions is very rare in primary care. Even if found to be effective, such time consuming interventions are unlikely to be taken up by GPs in sufficient numbers to produce a noticeable reduction in the occurrence of MDD. We found that many GPs offer empathic listening and reassurance. There is some evidence that non-directive counselling can be effective in reducing the onset of depression among primary care postpartum patients and further research should consider extending this avenue of inquiry (Holden et al., 1989).

\section{Strengths and limitations}

A strength of this study was its prospective design which allowed us to identify people with subthreshold depression at study entry and then document the care they subsequently received. This overcomes a limitation of retrospective studies in which patients are identified as subthreshold at study entry and treatments received previously are reported. Such studies will include individuals who, at study entry, are in partial remission from a recent major depressive episode. These individuals will have had a higher need for treatment during the recall period, and are more likely to have received treatment, than those with recent onset of symptoms. Therefore, 
estimates of care received by people with subthreshold depression are contaminated by the inclusion of level of care received by people with MDD.

A further strength was this study's ability to capture a wide range of GP responses for patients with subthreshold depression. Although the match between the six response categories and the NICE guidelines is imperfect, they approximate the stepped care approach outlined in the NICE guidelines more closely than has been done in any previous study.

A weakness of this study was the reliance of patient self-report and our inability to objectively validate receipt of individual interventions. Self-report is common in studies investigating treatment (Druss et al., 2007; Wang et al., 2005) however this methodology may introduce reporting bias. Although previous research indicates that as an individual's level of distress increases so too does their tendency to over-report their level of mental health treatment (Rhodes and Fung, 2004) it is also possible that self-report bias was in the opposite direction, i.e. individuals were receiving IPT and CBT but did not identify their treatment as such. Therefore, the degree to which patients may have received each intervention should be considered an estimate. Bias may also have been introduced because data came from patients who attended a GP who themselves agreed to participate in a research project on mental health. It is possible that GPs who participated in diamond were more likely than other GPs to be interested in mental health and / or were more likely to deliver higher quality care. As there is no centralised list in Australia recording whether a GP has a special interest in mental health it is difficult to estimate the representativeness of our sample in this regard. However, a 2005 study (Minas et al., 2005) investigating the impact of Australia's 'Better Outcomes in Mental Health Care Initiative' found that $25 \%$ of GPs who had not registered with the initiative and $60 \%$ of those that had registered were 'very interested' in mental health work. These figures suggest that the proportion of GPs in diamond with a special interest in mental health was not unduely high.

It must also be acknowledged that the data reported in this study was collected in 20052006. Therefore, the GP practices that are described in this paper may not capture advances in the management of depression in primary care that may have occurred since that time.

\section{Summary}

In summary, usual care of subthreshold depression in primary care varies widely. At one end of the spectrum, one fifth of people did not receive any mental health response over six months. At the other end of the spectrum, 27\% were prescribed antidepressants, which are not recommended for people with subthreshold symptoms and may have serious side-effects 
(Gotzche, 2014). Identifying the appropriate level of management for subthreshold depression was complicated by the finding that receipt of some mental health interventions was positively associated with the development of MDD, even when history of depression and symptom severity was taken into account. Future research should explore the development and use of a prognostic tool to identify people at risk of future MDD and explore the effectiveness of GP delivered non-directive counselling in preventing MDD among people with subthreshold depression. 


\section{References}

Ackermann, R., Williams, J., Jr., 2002. Rational treatment choices for non-major depressions in primary care. Journal of General Internal Medicine 17, 293-301.

Britt, H., Miller, G.C., Charles, J., Pan, Y., Valenti, L., Henderson, J., Bayram, C., O’Halloran, J., Knox, S., 2007. General practice activity in Australia 2005-06. General practice series no. 19. AIHW cat. no. GEP 19. Australian Institute of Health and Welfare., Canberra.

Cuijpers, P., Koole, S.L., van Dijke, A., Roca, M., Li, J., Reynolds, C.F., 2014. Psychotherapy for subclinical depression: meta-analysis. British Journal of Psychiatry 205, 268-274.

Cuijpers, P., Smit, F., 2004. Subthreshold depression as a risk indicator for major depressive disorder: a systematic review of prospective studies. Acta Psychiatrica Scandinavica 109, $325-331$.

Cuijpers, P., Smit, F., Van Straten, A., 2007. Psychological treatments of subthreshold depression: a meta-analytic review. Acta Psychiatrica Scandinavica 115, 434-441.

Dowrick, C.F., Frances, A., 2013. Medicalising unhappiness: new classification of depression risks more patients being put on drug treatment from which they will not benefit. British Medical Journal 347, 20-23.

Druss, B.G., Wang, P.S., Sampson, N.A., et al., 2007. Understanding mental health treatment in persons without mental diagnoses: Results from the national comorbidity survey replication. Archives of General Psychiatry 64, 1196-1203.

Gilchrist, G., Gunn, J., 2007. Observational studies of depression in primary care: What do we know? BMC Family Practice 8, 18.

Goldney, R.D., Fisher, L.J., Dal Grande, E., Taylor, A.W., 2004. Subsyndromal depression: Prevalence, use of health services and quality of life in an Australian population. Social Psychiatry \& Psychiatric Epidemiology 39, 293-298.

Gotzche, P.C., 2014. Why I think antidepressants do more harm than good. Lancet 1 104-106.

Gunn, J., Ayton, D., Densley, K., Pallant, J., Chondros, P., Herrman, H., Dowrick, C., 2012. The association between chronic illness, multimorbidity and depressive symptoms in an Australian primary care cohort. Soc Psychiatry Psychiatr Epidemiol 47, 175-184.

Gunn, J., Gilchrist, G., Chondros, P., Ramp, M., Hegarty, K., Blashki, G., Pond, D., Kyrios, M., Herrman, H., 2008. Who is identified when screening for depression in undertaken in general practice? Baseline findings from the diagnosis, management and outcomes of 
depression in primary care (Diamond) longitudinal study. Medical Journal of Australia $188,119-125$.

Harris, M.G., Hobbs, M., Burgess, P., Pirkis, J., Diminic, S., Siskind, D., Andrews, G., Whiteford, H., In press. Frequency and quality of mental health treatment for affective and anxiety disorders among Australian adults. Medical Journal of Australia.

Hermens, M.L.M., van Hout, H.P.J., Terluin, B., van der Windt, D.A.W.M., Beekman, A.T.F., van Dyck, R., de Haan, M., 2004. The prognosis of minor depression in the general population: a systematic review. General Hospital Psychiatry 26, 453-462.

Hodgson, R., Alwyn, T., John, B., Thom, B., Smith, A., 2002. The FAST Alcohol Screening Test. Alcohol and Alcoholism 37, 61-66.

Holden, J.M., Sagovsky, R., Cox, J.L., 1989. Counselling in a general practice setting: controlled study of health visitor intervention in treatment of postnatal depression. British Medical Journal 298, 223-226.

Karsten, J., Hartman, C.A., Smit, J.H., Zitman, F.G., Beekman, A.T.F., Cuijpers, P., Van der Does, A.J.W., Ormal, J., Nolen, W.A., Penninx, B.W., 2011. Psychiatric history and subthreshold symptoms as predictors of the occurrence of depressive or anxiety disorder within 2 years. British Journal of Psychiatry 198, 206.

Kessler, R.C., Ustun, T.B., 2004. The World Mental Health (WMH) Survey Initiative of the World Health Organization (WHO) Composite International Diagnostic Interview (CIDI). Internatinal Journal of Methods in Psychiatric Research 13, 93-121.

Kroenke, K., Spitzer, R.L., Williams, J.B.W., 2001. The PHQ-9: Validity of a brief depression severity measure. J GEN INTERN MED 16, 606-613.

Kurdyak, P.A., Gnam, W.H., 2005. Small signal, big noise: performance of the CIDI depression module. Canadian Journal Of Psychiatry. Revue Canadienne De Psychiatrie 50, 851-856.

Medicare Australia Statistics, https://www.medicareaustralia.gov.au/statistics/mbs_item.shtml.

Minas, I.H., Kilmidis, S., Kokanovic, R., 2005. Impact of Australia's 'Better Outcomes in Mental Health Care' initiative in Melbourne. Primary Care Mental Health 3, 111-121.

Morgan, A.J., Jorm, A.F., Mackinnon, A.J., 2012. Email-based promotion of self-help for subthreshold depression: Mood Memos randomised controlled trial. The British Journal of Psychiatry 200, 412-418.

National Institute for Health \& Clinical Excellence, 2010. The NICE guideline on the treatment and management of depression in adults (updated edition) London. 
Norbeck, J.S., 1984. Modification of Life Event Questionnaires for Use with Female Respondents. Research in Nursing \& Health 7, 61-71.

Pietrzak, R.H., Kinley, J., Afifi, T.O., Enns, M.W., Fawcett, J., Sareen, J., 2013. Subsyndromal depression in the United States: prevalence, course, and risk for incident psychiatric outcomes. Psychological Medicine 43, 1401-1414.

Radloff, L.S., 1977. The CES-D scale: A self-report depression scale for research in the general population. Applied Psychological Measurement 1, 385-401.

Rhodes, A.E., Fung, K., 2004. Self-reported use of mental health services versus administrative records: care to recall? International Journal of Methods in Psychiatric Research 13, 165 175.

Salas, M., Hotman, A., Stricker, B.H., 1999. Confounding by Indication: An Example of Variation in the Use of Epidemiologic Terminology. American Journal of Epidemiology 149, 981-983.

Sarason, I.G., Johnson, J.H., Siegal, J.M., 1978. Assessing the impact of life changes:

Development of the Life Experiences Survey. Journal of Consulting and Clinical Psychology 46, 932-946.

Simon, G., Von Korff, M., 1995 Recognition, Management, and Outcomes of Depression in Primary Care. Archives of Family Medicine 4, 99 - 105.

Spitzer, R.L., Kroenke, K., 1999. Instruction manual: Instructions for Patient Health Questionnaire (PHQ) and GAD-7 measures.

Wagner, H.R., Burns, B.J., Broadhead, W.E., Yarnall, K.S.H., Sigmon, A., Gaynes, B.N., 2000. Minor depression in family practice: functional morbidity, co-morbidity, service utilization and outcomes. Psychological Medicine 30, 1377-1390.

Wang, P.S., Lane, M., Olfson, M., Pincus, H.A., Wells, K.B., Kessler, R.C., 2005. Twelve-month use of mental health services in the united states: Results from the national comorbidity survey replication. Archives of General Psychiatry 62, 629-640.

Willemse, G.R.W.M., Smit, F., Cuijpers, P., Tiemens, B.G., 2004. Minimal-contact psychotherapy for sub-threshold depression in primary care: Randomised trial. British Journal of Psychiatry 185, 416-421.

Wittkampf, K.A., Naeije, L., Schene, A.H., Huyser, J., van Weert, H.C., 2007. Diagnostic accuracy of the mood module of the Patient Health Questionnaire: A systematic review. General Hospital Psychiatry 29, 388-395. 
Box 1 The NICE recommendations mapped to study measures

\begin{tabular}{|c|c|c|}
\hline \multicolumn{2}{|c|}{ NICE Guidelines } & \multirow{2}{*}{$\begin{array}{c}\text { diamond study } \\
\text { Data items }\end{array}$} \\
\hline Focus of the intervention & Nature of the intervention & \\
\hline & \multicolumn{2}{|c|}{ Sleep hygiene } \\
\hline People with depression. & $\begin{array}{l}\text { Offer advice on sleep hygiene if needed, } \\
\text { including: } \\
\text { - } \quad \text { Establishing regular sleep and wake times } \\
\text { - } \quad \text { Avoiding excess eating, smoking or drinking } \\
\text { alcohol before sleep } \\
\text { - } \quad \text { Creating a proper environment for sleep } \\
\text { - Taking regular physical exercise }\end{array}$ & $\begin{array}{l}\text { One of the following: } \\
-\quad \text { GP gave me advice on getting a good } \\
\text { night's sleep } \\
\text { - } \quad \text { Gave me advice on reducing drug or } \\
\text { alcohol intake } \\
\text { - } \quad \text { GP encouraged me to exercise } \\
\text { - } \quad \text { GP gave me advice on diet }\end{array}$ \\
\hline & \multicolumn{2}{|c|}{ Active monitoring } \\
\hline $\begin{array}{l}\text { For people who, in the } \\
\text { judgement of the } \\
\text { practitioner, may recover } \\
\text { with no formal intervention } \\
\text { or people with subthreshold } \\
\text { depressive symptoms who } \\
\text { request an intervention. }\end{array}$ & 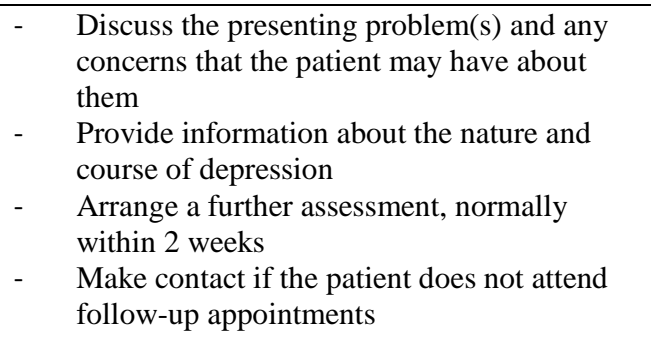 & $\begin{array}{l}\text { One of the following: } \\
-\quad \text { GP provided reassurance, } \\
\text { encouragement and explanation } \\
\text { - } \quad \text { GP gave me the chance to talk about } \\
\text { how I was feeling } \\
\text { - } \quad \text { GP gave me information (leaflets, } \\
\text { booklets, videos) about depression, } \\
\text { stress or worries } \\
\text { AND } \\
\text { - At least one GP visit in first } 3 \text { months }\end{array}$ \\
\hline & \multicolumn{2}{|c|}{ Low intensity psychosocial interventions } \\
\hline $\begin{array}{l}\text { People with persistent } \\
\text { subthreshold depressive } \\
\text { symptoms and patients with } \\
\text { subthreshold depressive } \\
\text { symptoms that complicate } \\
\text { the care of the chronic } \\
\text { physical health problem. }\end{array}$ & $\begin{array}{l}\text { Consider offering one or more of the following } \\
\text { interventions, guided by the patient's preference } \\
\text { - } \quad \text { A structured group physical activity } \\
\text { programme } \\
\text { - } \quad \text { A group-based peer support (self-help) } \\
\text { programme } \\
\text { - Individual guided self-help based on the } \\
\text { principles of cognitive behavioural therapy } \\
\text { (CBT) } \\
\text { - Computerised cognitive behavioural therapy } \\
\text { (CCBT). }\end{array}$ & 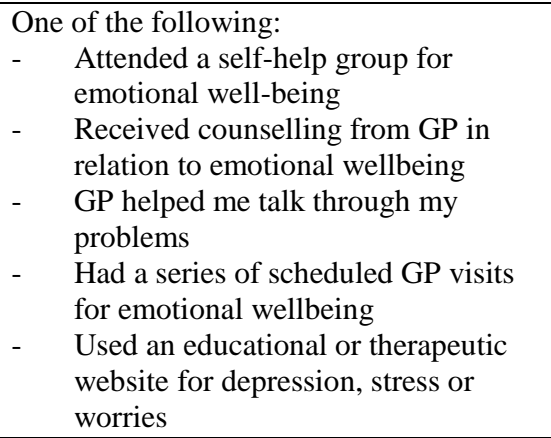 \\
\hline & \multicolumn{2}{|c|}{ High intensity psychosocial interventions } \\
\hline $\begin{array}{l}\text { For people with persistent } \\
\text { subthreshold depressive } \\
\text { symptoms or people who } \\
\text { have not benefited from a } \\
\text { low-intensity psychosocial } \\
\text { intervention. }\end{array}$ & $\begin{array}{l}\text { Cognitive Behaviour Therapy (CBT) or } \\
\text { Interpersonal Therapy (IPT) in combination with } \\
\text { following: } \\
\text { - The duration of treatment should typically } \\
\text { be in the range of } 16 \text { to } 20 \text { sessions over } 3 \text { to } \\
4 \text { months } \\
\text { As the aim of treatment is to obtain } \\
\text { significant improvement or remission the } \\
\text { duration of treatment may be reduced if } \\
\text { remission has been achieved }\end{array}$ & $\begin{array}{l}\text { One of the following: } \\
-\quad \text { Received counselling from GP in } \\
\text { relation to emotional wellbeing } \\
\text { - } \quad \text { GP helped me talk through my } \\
\text { problems } \\
\text { AND } \\
-\quad \geq 16 \text { visits to a GP }\end{array}$ \\
\hline & \multicolumn{2}{|c|}{ Pharmacotherapy } \\
\hline $\begin{array}{l}\text { People with a past history } \\
\text { of moderate or severe } \\
\text { depression or initial } \\
\text { presentation of subthreshold } \\
\text { depressive symptoms that } \\
\text { have been present for a long } \\
\text { period or subthreshold } \\
\text { depressive symptoms that } \\
\text { persist after other } \\
\text { interventions. }\end{array}$ & $\begin{array}{l}\text { Do not use antidepressants routinely to treat } \\
\text { persistent subthreshold depressive symptoms or } \\
\text { mild depression because the risk-benefit ratio is } \\
\text { poor }\end{array}$ & $\begin{array}{l}\text { - GP prescribed antidepressants during } \\
\text { follow-up }\end{array}$ \\
\hline & \multicolumn{2}{|l|}{ Referral } \\
\hline $\begin{array}{l}\text { For people with severe } \\
\text { depression and those with } \\
\text { moderate depression and } \\
\text { complex problems }\end{array}$ & $\begin{array}{l}\text { Refer to specialist mental health services for a } \\
\text { programme of coordinated multiprofessional care }\end{array}$ & $\begin{array}{l}\text { GP referred me to another health } \\
\text { professional for my emotional } \\
\text { wellbeing }\end{array}$ \\
\hline
\end{tabular}


Table 1 Baseline patient sociodemographic and clinical characteristics

\begin{tabular}{lcccccc}
\hline & Total & $\begin{array}{c}\text { No depression } \\
\text { history } \\
\mathbf{n = 1 1 8}\end{array}$ & $\begin{array}{c}\text { History of } \\
\text { depression } \\
\mathbf{n = 1 3 2}\end{array}$ & & & \\
& Mean (s.d.) & Mean (s.d.) & Mean (s.d.) & Co-eff. & $\mathbf{9 5 \%} \mathbf{C I}$ & $\boldsymbol{p}$ \\
\hline Depression severity & $7.1(3.6)$ & $6.5(3.6)$ & $7.7(3.5)$ & 1.18 & $0.37-2.00$ & 0.006 \\
Age & $50.3(13.1)$ & $50.2(13.5)$ & $50.5(12.7)$ & 0.27 & $-3.80-4.35$ & 0.89 \\
\hline & $\mathrm{n}(\%)$ & $\mathrm{n}(\%)$ & $\mathrm{n}(\%)$ & $\mathbf{O R}^{*}$ & $\mathbf{9 5 \%} \mathbf{C I}$ & $\boldsymbol{p}$ \\
\hline Female, & $175(70.0)$ & $74(62.7)$ & $101(76.5)$ & 1.97 & $1.15-3.39$ & 0.01 \\
Married & $118(47.4)$ & $62(52.5)$ & $56(42.8)$ & 0.64 & $0.41-1.00$ & 0.05 \\
Relies on pension & $95(38.0)$ & $34(28.8)$ & $61(46.2)$ & 2.03 & $1.20-3.43$ & 0.008 \\
Education: $\leq$ year 10 & $35(14.1)$ & $11(9.5)$ & $24(18.2)$ & 1.95 & $0.96-3.95$ & 0.06 \\
Lives alone & $50(20.0)$ & $15(12.7)$ & $35(26.5)$ & 2.62 & $1.30-5.25$ & 0.007 \\
Negative life event & $190(76.0)$ & $91(77.1)$ & $99(75.0)$ & 0.89 & $0.49-1.60$ & 0.70 \\
Positive life event & $74(29.6)$ & $38(32.2)$ & $36(27.3)$ & 0.79 & $0.43-1.43$ & 0.44 \\
Chronic illness & $175(70.0)$ & $84(71.2)$ & $91(68.9)$ & 0.94 & $0.55-1.59$ & 0.81 \\
Smoking & $61(24.5)$ & $26(22.0)$ & $35(26.7)$ & 1.24 & $0.62-2.46$ & 0.53 \\
Hazardous drinking & $64(25.7)$ & $34(29.1)$ & $30(22.7)$ & 0.69 & $0.35-1.34$ & 0.28 \\
Anxiety disorder & $40(16.2)$ & $16(13.6)$ & $24(18.6)$ & 1.45 & $0.84-2.48$ & 0.17 \\
Suicidal ideation & $49(19.7)$ & $20(17.0)$ & $29(22.1)$ & 1.39 & $0.73-2.64$ & 0.30 \\
\hline
\end{tabular}

\# Denominators may vary due to missing data

* Reference group = No history 
Table 2 Visits to GPs and other mental health professionals over 6 months

\begin{tabular}{|c|c|c|c|c|c|c|}
\hline & 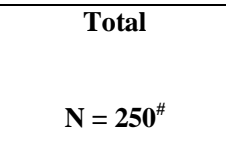 & $\begin{array}{c}\text { No depression } \\
\text { history } \\
\mathbf{n}=\mathbf{1 1 8}\end{array}$ & $\begin{array}{c}\text { History of } \\
\text { depression } \\
n=132\end{array}$ & & & \\
\hline Number of & $\mathrm{n}(\%)$ range & $\mathrm{n}(\%)$ range & $\mathrm{n}(\%)$ range & IRR $^{*}$ & $95 \% \mathrm{CI}$ & $p$ \\
\hline GP visits & $4.72(4.22) 0-24$ & $4.10(4.05) 0-23$ & $5.27(4.30) 0-24$ & 1.28 & $1.10-1.49$ & $<0.001$ \\
\hline Different GPs visited & $2.12(1.41) 0-8$ & 1.93 (1.32) 0-6 & $2.28(1.46) 0-8$ & 1.18 & $0.94-1.48$ & 0.05 \\
\hline Any visit to & $\mathrm{n}(\%)$ & $\mathrm{n}(\%)$ & $\mathrm{n}(\%)$ & OR & $95 \% \mathrm{CI}$ & $p$ \\
\hline Psychologist/Counsellor & $27(10.8)$ & $10(8.5)$ & $17(12.9)$ & 1.80 & $0.72-4.46$ & 0.20 \\
\hline Psychiatrist & $16(6.4)$ & $3(2.5)$ & $13(10.0)$ & 4.43 & $1.14-17.18$ & 0.03 \\
\hline
\end{tabular}

\# Denominators may vary due to missing data

* Reference group $=$ No history 
Table 3 Mental health interventions received over 6 months

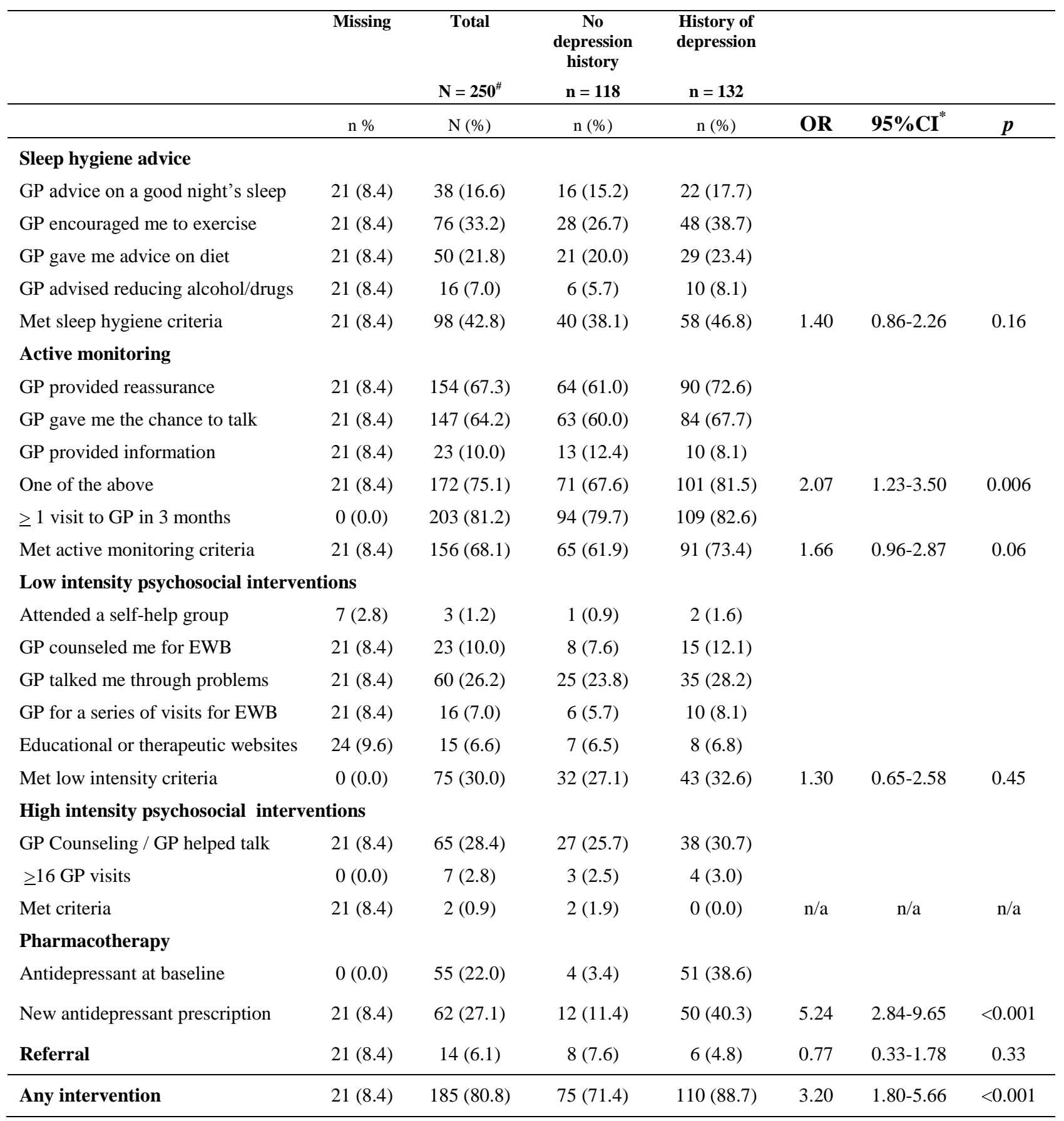

\# Denominators may vary due to missing data

* Reference group = No history 
Table 4 Association between baseline patient characteristics and developing MDD over six months

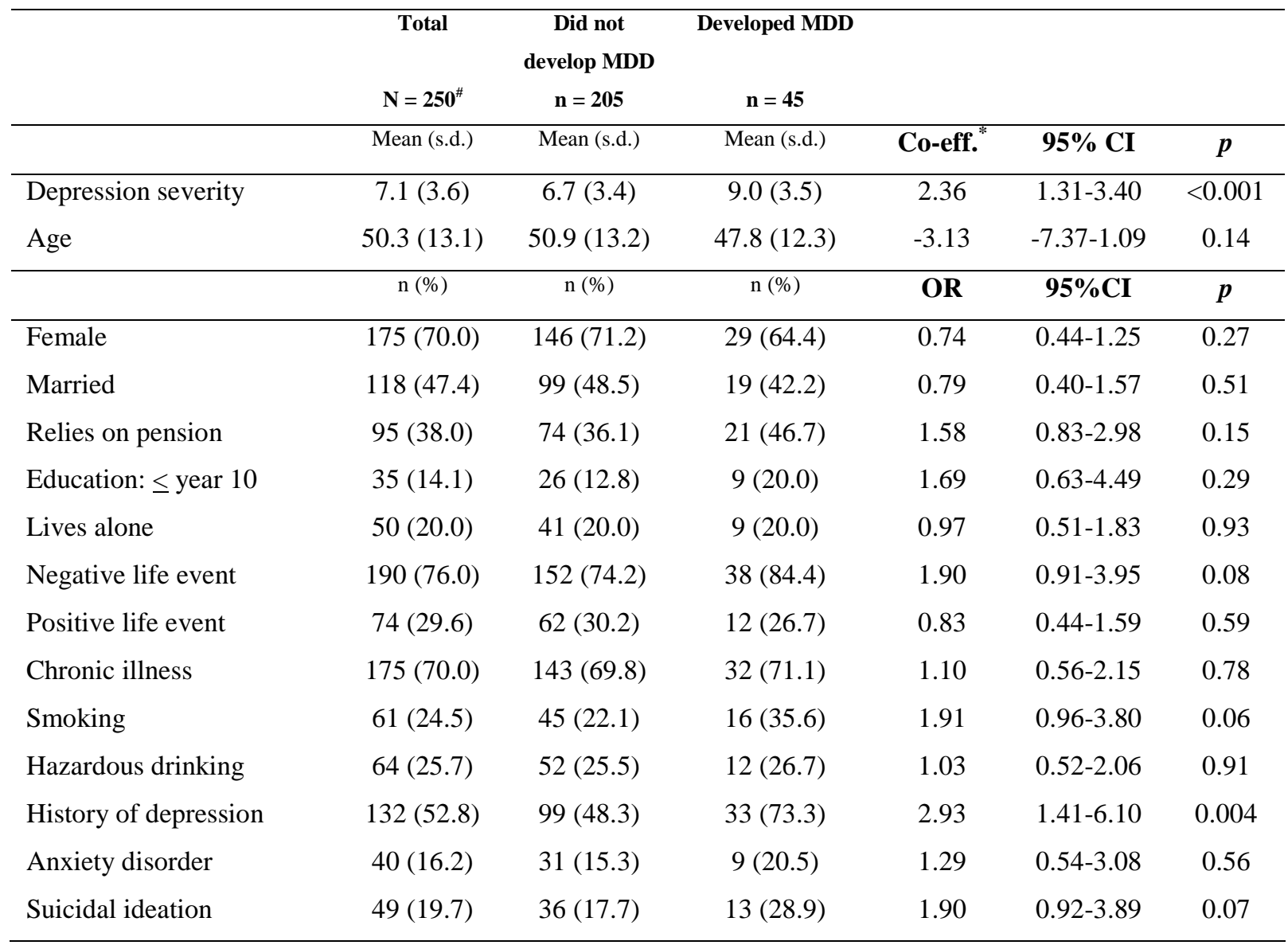

\# Denominators may vary due to missing data

* Reference group $=$ No history 
Table 5 Association between receiving a mental health intervention and developing MDD over six months

\begin{tabular}{|c|c|c|c|c|c|c|c|}
\hline & Total & No MDD & $\begin{array}{c}\text { Developed } \\
\text { MDD }\end{array}$ & Unadjusted & & Adjusted* & \\
\hline & $\mathrm{N}=\mathbf{2 5 0}^{\#}$ & $\mathrm{n}=\mathbf{2 0 5}$ & $n=45$ & & & & \\
\hline & $\mathrm{n}(\%)$ & $\mathrm{n}(\%)^{\#}$ & $\mathrm{n}(\%)^{\#}$ & OR 95\% CI & $p$ & OR $95 \%$ CI & $p$ \\
\hline Sleep hygiene advice & $98(42.8)$ & $69(36.9)$ & $29(69.1)$ & $3.80(1.96-7.37)$ & $<0.001$ & $3.04(1.56-5.94)$ & 0.001 \\
\hline Active monitoring & $156(68.1)$ & $121(64.7)$ & $35(83.3)$ & $2.76(1.12-6.78)$ & 0.02 & $2.41(0.95-6.15)$ & 0.06 \\
\hline Low-intensity psych & $75(30.0)$ & $57(27.8)$ & $18(40.0)$ & $1.75(0.91-3.34)$ & 0.09 & $1.42(0.72-2.78)$ & 0.30 \\
\hline Pharmacotherapy & $62(27.1)$ & 41 (21.9) & $21(50.0)$ & $3.65(1.73-7.72)$ & 0.001 & $2.48(1.12-5.51)$ & 0.02 \\
\hline Referral & $14(6.1)$ & $10(5.4)$ & $4(9.5)$ & $1.88(0.38-9.28)$ & 0.43 & $2.22(0.37-13.23)$ & 0.38 \\
\hline
\end{tabular}

\# Denominators may vary due to missing data

* Adjusted for history of depression and baseline depression severity 


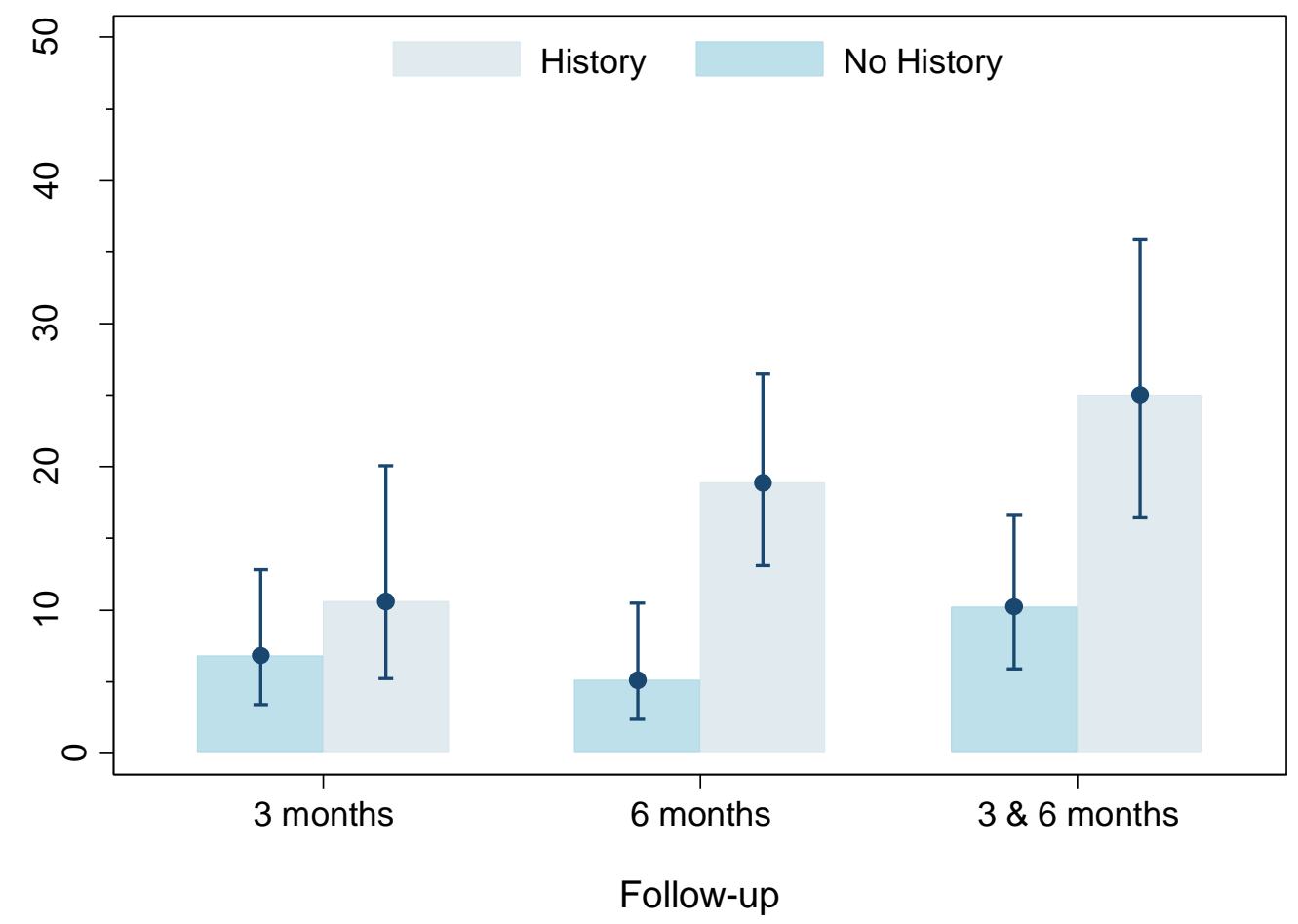

Figure $1 \mathrm{MDD}$ at three and six months and over total follow-up by history of depression 


\section{University Library}

\section{- M M N E R VA A gateway to Melbourne's research publications}

Minerva Access is the Institutional Repository of The University of Melbourne

Author/s:

Davidson, SK;Harris, MG;Dowrick, CF;Wachtler, CA;Pirkis, J;Gunn, JM

Title:

Mental health interventions and future major depression among primary care patients with subthreshold depression

Date:

2015-05-15

Citation:

Davidson, S. K., Harris, M. G., Dowrick, C. F., Wachtler, C. A., Pirkis, J. \& Gunn, J. M. (2015). Mental health interventions and future major depression among primary care patients with subthreshold depression. JOURNAL OF AFFECTIVE DISORDERS, 177, pp.65-73. https:// doi.org/10.1016/j.jad.2015.02.014.

Persistent Link:

http://hdl.handle.net/11343/58463 